\title{
Peculiarities of High Power Infrared Detection on Narrow-Gap Semiconductor $\boldsymbol{p}-\boldsymbol{n}$ Junctions
}

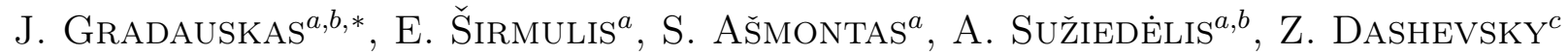 \\ AND V. KASIYAN ${ }^{c}$
}

${ }^{a}$ Semiconductor Physics Institute of the Center for Physical Sciences and Technology

A. Goštauto 11, Vilnius 01108, Lithuania

${ }^{b}$ Vilnius Gediminas Technical University, Saulètekio av. 11, Vilnius 10223, Lithuania

${ }^{c}$ Department of Materials Engineering, Ben-Gurion University of the Negev, Beer-Sheva 84105, Israel

\begin{abstract}
We report on experimental results of photosignal investigation in $\mathrm{HgCdTe}$, InSb and $\mathrm{PbTe}$ semiconductor $p-n$ junctions under the action of an intense pulsed $\mathrm{CO}_{2}$ laser. The influence of laser power, external bias voltage as well as the diode temperature on the photosignal formation has been studied. We show that under certain conditions both classical photovoltaic and hot carrier phenomena may act simultaneously within a junction thus strongly impacting on responsivity and speed of operation of the device. The drawn conclusions may be of great importance for the development of high power optoelectronic devices.
\end{abstract}

PACS: 78.56.-a, 78.70.-g, 84.60.Jt, 85.60.Dw

\section{Introduction}

Classical photovoltage appearing on an illuminated semiconductor $p-n$ junction is induced by electron-hole pair generation and their separation in the potential field of the junction. The fundamental requirement for the operation of such a detector is the incident radiation energy to be higher than a semiconductor bandgap.

Diodes with a $p-n$ junction remain the most important infrared (IR) detectors despite numerous attempts to replace them with the Schottky barriers, multiple quantum wells and quantum dots, strain layer superlattices and alternative materials such as high temperature superconductors or pyroelectric detectors [1-6]. HgCdTe, InSb and $\mathrm{PbTe}$ semiconductor diodes as intrinsic detectors are widely used to cover the wavelengths of the atmospheric windows $3-5 \mu \mathrm{m}$ and 8-14 $\mu \mathrm{m}[7]$.

On the other hand, a wide-gap semiconductor $p-n$ junction illuminated with IR laser light having photon energy lower than the bandgap produces a photovoltage resulting from the free carrier absorption [8-11]. In this case free carriers obtain additional energy and by overcoming the potential barrier of the junction form the so-called hot carrier photovoltage. Formation of this photovoltage has been investigated on relatively wide-gap semiconductors' Ge [8-10], Si [11, 12], GaAs [13, 14] $p-n$ junctions illuminated with a $\mathrm{CO}_{2}$ laser (wavelength $10.6 \mu \mathrm{m}$ )

\footnotetext{
* corresponding author; e-mail: jonas@pfi.lt
}

radiation. Polarity of the hot carrier photovoltage is opposite to that of a classical one.

Obviously, if a narrow-gap semiconductor is illuminated with a light with photon energy satisfying conditions for interband absorption, still the intraband (free carrier) absorption may take action simultaneously. It was established that photoconductivity in $\mathrm{HgCdTe}$ may be influenced by the free carrier heating even under a moderate intensity IR laser light [15]. Studies of two-photon interband absorption coefficient in InSb have revealed influence of free carrier absorption on its value [16]. Therefore it is important to find methods clarifying and distinguishing both mentioned absorption mechanisms which determine detection properties of semiconductor diodes.

In this paper we report a photosignal on $\mathrm{HgCdTe}, \mathrm{InSb}$ and $\mathrm{PbTe} p-n$ junctions under pulsed $\mathrm{CO}_{2}$ laser excitation and show presence of both interband and intraband absorption in these semiconductor structures.

\section{Experimental}

$\mathrm{Hg}_{0.8} \mathrm{Cd}_{0.2} \mathrm{Te}$ photodiodes were prepared by arsenic diffusion from the vapor source into $n$-type single crystal substrates followed by annealing process under $\mathrm{Hg}$ -saturation condition $\left(n \approx 1 \div 6 \times 10^{15} \mathrm{~cm}^{-3}\right.$ at $\left.77 \mathrm{~K}\right)$.

$\mathrm{InSb}$ photodiodes were prepared by liquid-phase epitaxy deposition of $p$-type $10 \mu \mathrm{m}$ thick layer on $n$ -substrate $\left(n=2 \times 10^{14} \mathrm{~cm}^{-3}\right.$ and $p \approx 10^{16} \mathrm{~cm}^{-3}$ at $77 \mathrm{~K})$. 
PbTe $p-n$ structures were formed by $\mathrm{In}_{4} \mathrm{Te}_{3}$ gas diffusion into a $p$-substrate at $\approx 70 \mu \mathrm{m}$ depth $(n \approx 6 \times$ $10^{18} \mathrm{~cm}^{-3}$ and $\left.p \approx 10^{18} \mathrm{~cm}^{-3}\right)$. Mask pattern with $50 \times 50 \mu \mathrm{m}^{2}$ openings was used to form the diode structures by subsequent mesa etching.

As the IR radiation source the passively Q-switched $\mathrm{CO}_{2}$ laser producing $150 \mathrm{~ns}$ duration pulses of $10.6 \mu \mathrm{m}$ wavelength was used. Maximum laser intensity reached $1 \mathrm{MW} / \mathrm{cm}^{2}$. The laser pulse shape was monitored with Ge photon-drag detector; the intensity of the light was varied with neutral teflon filters. All the experiments were performed at liquid nitrogen temperature.

\section{Results}

\subsection{Radiation power effect}

At low intensities $\mathrm{CO}_{2}$ laser radiation induces the classical photovoltage across the $\operatorname{HgCdTe} p-n$ junction (see Fig. 1, open dots; trace $a$ in the inset). The signal linearly increases in magnitude with the radiation intensity, and at high excitation levels starts saturating. The saturation is related with the potential barrier height of a $p-n$ junction. Further increase of the radiation intensity causes raise of another signal component of opposite polarity (Fig. 1, solid dots; trace $b$ in the inset). This component is fast, it perfectly follows temporal shape of the wavy laser pulse and its magnitude also linearly increases with the intensity. All three facts: polarity, linear course and fast speed, indicate the second component to be the hot carrier photovoltage. This photovoltage is much faster than the classical one since the speed of it is conditioned by the carrier energy relaxation time (which is usually of the order of tens of picoseconds in semiconductors), while the temporal behavior of the classical photovoltage is determined by the excess carrier lifetime (about $500 \mathrm{~ns}$ in our case).

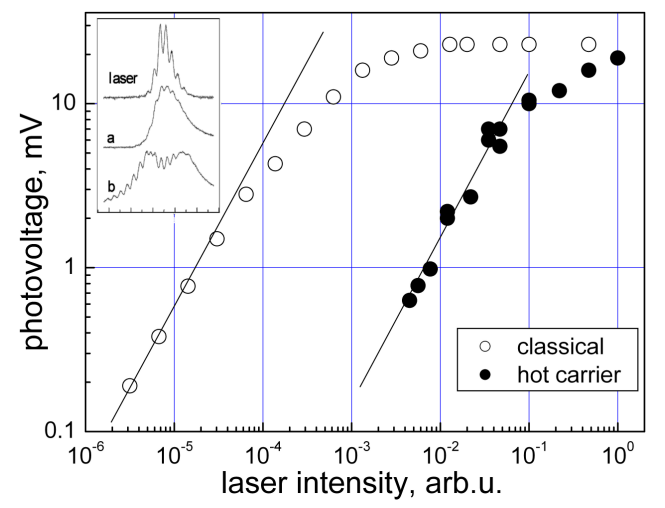

Fig. 1. Photovoltage across $\mathrm{HgCdTe} p-n$ junction against laser intensity. Straight lines represent linear dependence. Inset: wavy laser pulse and photovoltage pulse shape at lower $(a)$ and higher $(b)$ radiation intensities.

A very similar picture is observed in the dependence of photosignal detected across the InSb $p-n$ junction on the laser intensity (Fig. 2). The main difference is that the classical photovoltage in this case originates from the two-photon absorption of the $\mathrm{CO}_{2}$ laser radiation and therefore follows the square law $[16,17]$.

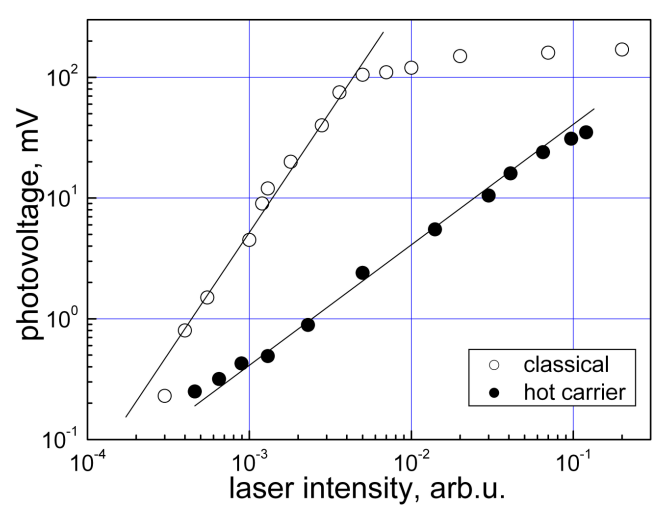

Fig. 2. Photovoltage across InSb $p-n$ junction against laser intensity. Straight lines represent square (left) and linear (right) dependences.

It is worth noting that at high enough laser radiation intensities the classical and the hot carrier photovoltages, which are of opposite polarities, can reach equal values of magnitude (see Fig. 1 and Fig. 2).

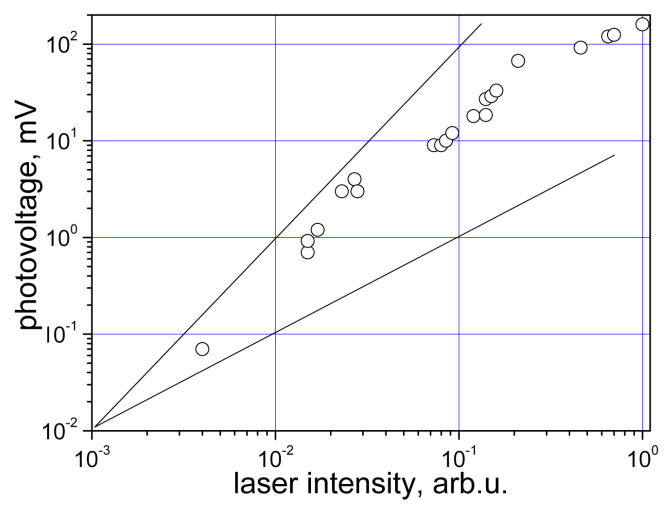

Fig. 3. Photovoltage across $\mathrm{PbTe} p-n$ junction against laser intensity. Straight lines represent square (left) and linear (right) dependences.

Some different behavior is demonstrated by the $\mathrm{PbTe}$ $p-n$ junction. Illumination of it with the laser radiation also gives rise to the photovoltage with polarity corresponding to the classical one. The signal also tends to saturate at high laser light intensities (Fig. 3). However, the dependence is neither linear nor square. Earlier we have revealed evidence of the two-photon absorption of the $\mathrm{CO}_{2}$ laser radiation in PbTe Schottky diodes [18] and close-to-surface $p-n$ junctions [19] at liquid nitrogen temperature $(0.22 \mathrm{eV}$ of the forbidden energy gap against $0.117 \mathrm{eV}$ of the photon energy at $80 \mathrm{~K}$ ). On the other hand, it was declared that due to very strong free carrier absorption of the laser radiation the photosignal in 
the deeply formed $p-n$ junction may result from absorption on free carriers, heat transfer from the hot electrons to the photon system, thermal generation of electronhole pairs and consequently their separation in the built-in electric field of the junction [20]. Since in our case the index of the photosignal dependence on the laser intensity is about 1.4, we state that both phenomena, i.e. two-photon absorption and free carrier absorption, are responsible for the photovoltage formation. In contrast to the previous $\mathrm{HgCdTe}$ and $\mathrm{InSb}$ cases, pure hot carrier photovoltage has not been detected due to the relatively deeply buried junction.

\subsection{Voltage bias effect}

If the measurements are performed in the closed circuit regime, the classical or (and) hot carrier photocurrent flows across the $p-n$ junction. The strength of the first one depends on the applied bias voltage $U$ through the width of the depletion region $W[21]$ :

$$
j_{\text {ph-class }} \propto W \propto\left(V_{0}-U\right)^{1 / 2},
$$

where $V_{0}$ is the built-in potential barrier of the junction. Whereas the second one obeys the exponential dependence [14]:

$$
j_{\mathrm{ph}-\mathrm{hot}} \propto \exp (U) \text {. }
$$

it means that the forward bias voltage applied on a $p-n$ junction increases the hot carrier photocurrent, while the reverse one is favorable for the flow of the classical photocurrent. Photosignal across the InSb diode traces perfectly illustrate this consideration (Fig. 4). It is seen that at high enough forward bias voltage values the photosignal may consist totally of the hot carrier component (Fig. 4b, trace 5).

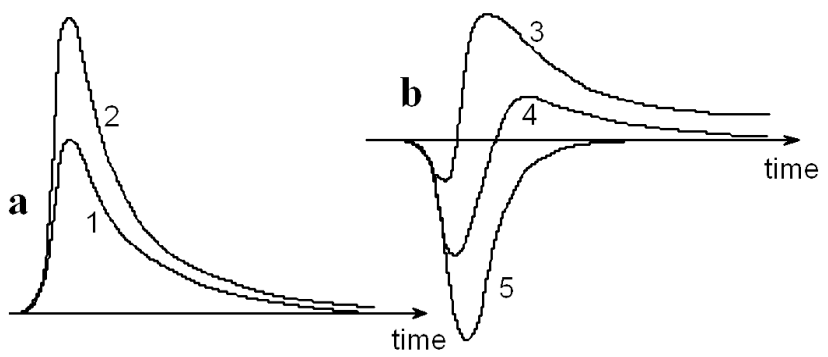

Fig. 4. Traces of photosignal pulse induced across InSb $p-n$ junction at zero and reverse bias (a) and at forward (b) bias voltage: $U=0 \mathrm{~V}(1),-0.20 \mathrm{~V}(2), 0.167 \mathrm{~V}(3)$, $0.20 \mathrm{~V}(4), 0.227 \mathrm{~V}(5)$. Positive polarity corresponds to the classical photovoltage, negative shows hot carrier photovoltage.

Moreover, it should be noted that the photovoltage itself biases the junction, e.g. the generated classical photovoltage biases the junction in a forward direction thus favoring the emergence of the hot carrier photovoltage, and vice versa.

\subsection{Temperature effect}

Obviously, the classical photovoltage decreases with increasing temperature due to decreasing barrier height. We have witnessed this correlation in the InSb junctions. The same conclusion has been drawn from the investigations of photosignal across the $\mathrm{PbTe} p-n$ junction [20]. However, in the view of the increase of lead telluride forbidden energy gap with temperature, the two-photon absorption induced photovoltage can be generated only up to the $110 \mathrm{~K}$ temperature when the gap becomes equal to doubled energy of the $\mathrm{CO}_{2}$ laser radiation photon (if the Moss-Burstein effect is not taken into account).

As for the hot carrier photovoltage, the temperature increase encumbers its formation mainly due to the decrease of carrier mobility.

\section{Conclusions}

We have experimentally shown that detection quality of detectors having a narrow-gap semiconductor $p-n$ junction and operating in the intrinsic mode can be significantly influenced by the free carrier absorption phenomenon. In $\mathrm{HgCdTe}$ and InSb cases, at high enough power of laser radiation the hot carrier photovoltage magnitude reaches the one of the classical photovoltage thus reducing the responsivity of the detectors. Moreover, the applied external or optically induced forward bias voltage also favors rise of the hot carrier photosignal. PbTe case shows that the influence of free carrier absorption on the photosignal formation may be conditioned by the depth of the $p-n$ junction from the surface. Rise of the detector operation temperature hinders both classical and hot carrier photosignal formation.

\section{References}

[1] E.L. Dereniak, G.D. Boreman, Infrared Detectors and Systems, Wiley-Interscience, New York 1996.

[2] S. Chakrabarti, A.D. Stiff-Roberts, X.H. Su, P. Bhattacharya, G. Ariyawansa, A.G.U. Perera, J. Phys. D, Appl. Phys. 38, 2135 (2005).

[3] G. Bishop, E. Plisa, J.B. Rodriguez, Y.D. Sharma, H.S. Kim, L.R. Dawson, S. Krishna, J. Vac. Sci. Technol. B 26, 1145 (2008).

[4] J.M. Gildemeister, A.T. Lee, P.L. Richards, Appl. Phys. Lett. 77, 4040 (2000).

[5] M. Lindgren, M. Currie, C. Williams, T.Y. Hsiang, P.M. Fauchet, R. Sobolewski, S.H. Moffat, R.A. Hughes, J.S. Preston, F.A. Hegmann, IEEE Trans. Appl. Supercond. 7, 3422 (1997).

[6] M. Shankar, J.B. Burchett, Q. Hao, B.D. Guenther, D.J. Brady, Opt. Eng. 45, 106401 (2006).

[7] A. Rogalski, K. Adamiec, J. Rutkowski, Narrow-Gap Semiconductor Photodiodes, SPIE Press Book, Bellingham 2000.

[8] M. Umeno, Y. Sugito, T. Jimbo, H. Hattori, Y. Ameniya, Solid State Electron. 21, 191 (1978).

[9] S. Ašmontas, E. Širmulis, S. Stonys, Sov. Phys.-Collection 24, 76 (1984). 
[10] S. Ašmontas, E. Maldutis, E. Širmulis, Int. J. Optoelectron. 3, 263 (1988).

[11] S. Ašmontas, J. Gradauskas, E. Širmulis, Proc. SPIE 1512, 131 (1991).

[12] M. Lopez Saenz, J.M. Guerra Perez, Appl. Phys. Lett. 68, 675 (1996).

[13] S. Ašmontas, J. Gradauskas, D. Seliuta, A. Šilènas, E. Širmulis, I.Ya. Marmur, Proc. SPIE 3093, 35 (1996).

[14] S. Ašmontas, J. Gradauskas, D. Seliuta, E. Širmulis, Proc. SPIE 4423, 18 (2001).

[15] W. Weili, X. Qijiang, Chin. J. Infrared Millimeter Waves 6, 439 (1987).

[16] A.F. Gibson, C.B. Hatch, P.N.D. Maggs, D.R. Tilley, A.C. Walker, J. Phys. C 9, 3259 (1976).
[17] V. Nathan, A.H. Guenther, S.S. Mitra, J. Opt. Soc. Am. B 16, 294 (1985).

[18] S. Ašmontas, Z. Dashevsky, M.P. Dariel, J. Gradauskas, A. Jarashneli, S. Shusterman, A. Sužiedèlis, G. Valušis, Proc. SPIE 4318, 230 (2001).

[19] S. Ašmontas, L. Vingelis, J. Gradauskas, Z. Dashevskii, A. Kasiyan, Thermoelectricity 1, 54 (1995).

[20] Z. Dashevsky, V. Kasiyan, S. Asmontas, J. Gradauskas, E. Shirmulis, E. Flitsiyan, L. Chernyak, J. Appl. Phys. 106, 076105 (2009).

[21] B.G. Streetman, S. Banerjee, Solid State Electronic Devices, Prentice Hall, New Jersey 2000. 